\title{
A comparison of chinchilla auditory evoked response and behavioral response thresholds'
}

\author{
DONALD HENDERSON, ${ }^{2}$ SHINJIRO ONISHI ${ }^{3}$ DONALD H. ELDREDGE, AND HALLOWELL DAVIS \\ CENTRAL INSTITUTE FOR THE DEAF
}

The auditory sensitivity of the chinchilla was measured by both a behavioral and auditory evoked-response (AER) procedure There was no significant difference in either the waveform or the threshold of the $A E R$ recorded over the tentorium from awake and anesthetized chinchillas. The chinchilla threshold for pure tones appears to be more sensitive when measured by the behavioral technique than by the AER method. Both the AER and behavioral response of three chinchillas exposed to a $105 \mathrm{dBSPL}$ octave band of notse centered at $2 \mathrm{KHz}$ for $3 \mathrm{~h}$ shifted markedly after the exposure. However, the difference between the $A E R$ and behavioral response threshold was reduced or absent. During the process of recovery the difference between the two responses begins to reappear

Rothenberg and Davis (1967) have recently reported an early auditory evoked-response (AER) that can be recorded from the anesthetized chinchilla. The threshold for the AER can be recorded to stimuli that are in the range of the chinchilla's behavioral threshold. The chinchilla evoked response that was reported differs from the human AER that is typically used in audiometry for threshold measurement. The human AER is a slow diffuse response and is characterized by an initial negative wave. The response is best recorded at the vertex, and has been named the V-potential. The V-potential has an amplitude of approximately $5 \mu \mathrm{V}$ for signals of $30 \mathrm{~dB}$ sensation level and the latency of the response is about $100 \mathrm{msec}$ after the stimulus onset. The chinchilla AER reported by Rothenberg and Davis was recorded over the tentorium by a subcutancous needle electrode. The response is an early positive wave of approximately $5 \mu \mathrm{V}$ followed by a slower and more variable negative wave. The latency of the first positive peak is 6 to $8 \mathrm{msec}$ after the stimulus onset.

The chinchilla AER is potentially useful for threshold testings because it offers an alternative to the time-consuming and inherently complicated behavioral methods. However, the justification for extrapolation from the AER to the behavioral threshold rests on the demonstration that these two classes of response covary.

The following set of experiments is directed at answering these questions: (1) Can the AER be recorded from an awake chinchilla and, if so, (2) are there any differences between the AER recorded from awake and anesthetized animals, (3) does the AER threshold correlate with the behavioral threshold, and (4) when the behavioral threshold is elevated by noise exposure, does the AER threshold change proportionally?

\section{Behavioral Testing}

\section{METHOD}

The Ss were three male chinchillas between 400 and $550 \mathrm{~g}$ weight. Each $S$ was made monaural by the destruction of its left cochlea. Normal behavioral thresholds were determined by the conditioned-avoidance technique developed for cats by Miller, Watson, and Covell (1963) and later modified by Peters (1965) for chinchillas. The psychophysical method was a modified method of limits which began with a descending series having a step-interval of 10 to $20 \mathrm{~dB}$; the termination of this series was followed by an ascending step of $10 \mathrm{~dB}$. A threshold is defined as the intensity value half-way between the lowest "hit" and highest "miss" at each intensity. The test stimulus consisted of three tonal pulses separated by silent intervals of $500 \mathrm{msec}$. Each pulse has a $50-\mathrm{msec}$ rise, $650-\mathrm{msec}$ plateau and a $50-\mathrm{msec}$ fall.

\section{Surgical Procedures}

Each S was anesthetized by a light dose of dial in urethane. The skull and bulla were exposed and a hole was made in the bulla for access to the round window. One active electrode (Lukens 50 monofilament stainless steel) was placed next to the round window, a second active electrode on the dura over the tentorium, and an indifferent reference electrode in the nasal cavity. The electrodes were connected to a three-pole transistor plug by silver-impregnated epoxy and the assembly was fastened to the skull with dental acrylic (see Fig. 1). The operation was done with sterile techniques including the placement and sealing of the round window electrode. The responses from the round window were difficult to record reliably because the electrode was not stabilized properly. The Ss were given a daily prophylactic dose of Rondomycin for five days after the operation. A male connector was inserted in the plug during a recording period, and $\mathrm{S}$ was grounded with a Grass silver cup electrode on the ear.

\section{Acoustic Signals}

The AER data were collected in either the EEG Laboratory or the Physiology Laboratory of the Central Institute for the Deaf. The stimulus and recording system in the EEG Laboratory has been described in Davis et al (1966). The stimuli were tone pips produced by ringing an Allison 2 BR bandpass filter with a rectangular pulse. The high-pass and low-pass dials of the filter were set to the same nominal frequency $(600,1200,2400$, and 4800 ) and the pass band was expanded by $1 / 10$ of the nominal

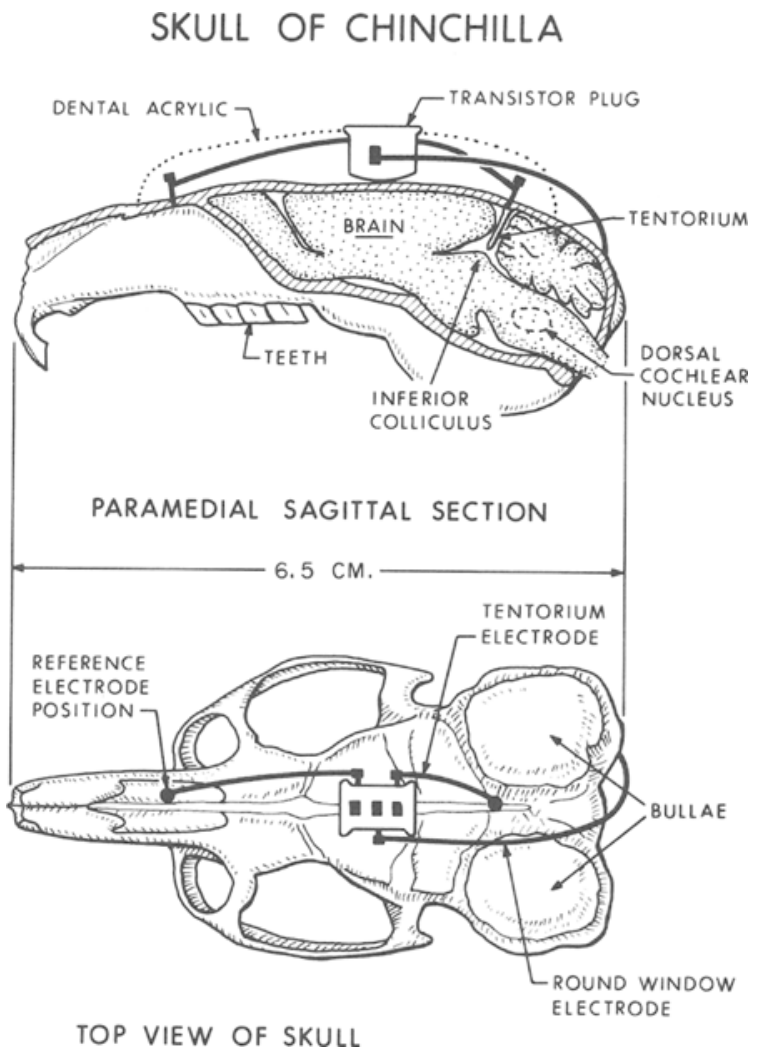

Fig. 1. Major neural structures of the chinchilla and external landmarks. The electrode positions and transistor plug connections are shown. 


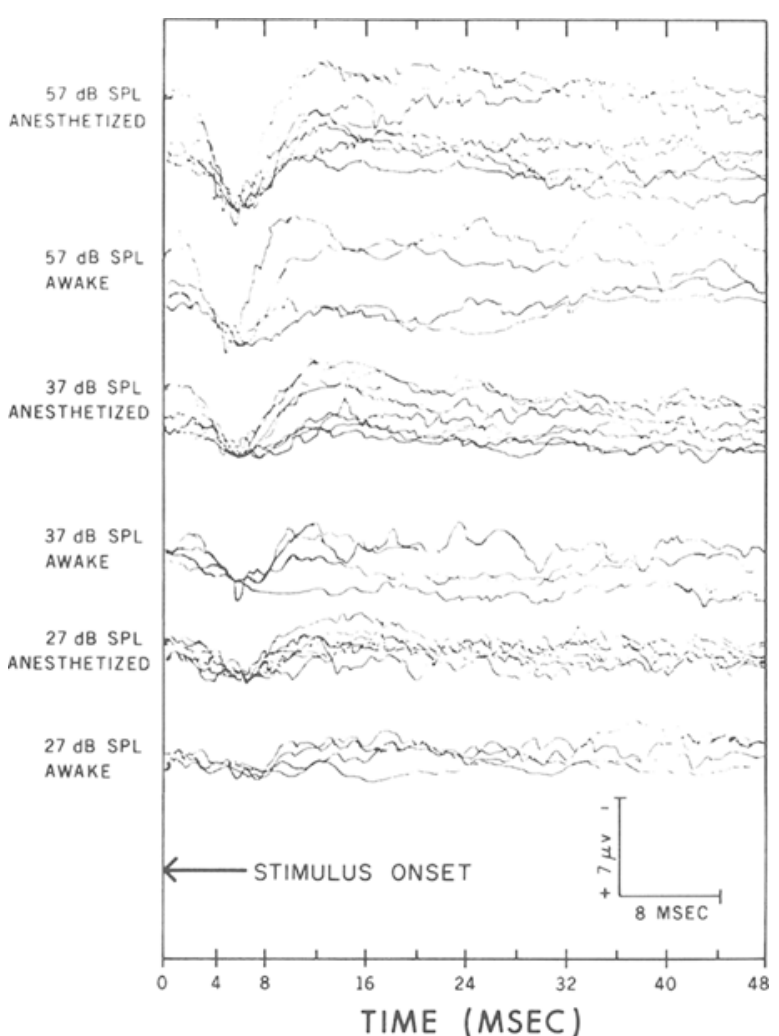

Fig. 2. AERs recorded from anesthetized and conscious chinchillas.

frequency by vernier adjustments. The duration of the pulse was set for each frequency to yield the smoothest waveform and the maximum voltage. The tone pips reached their maximum voltage during the third cycle and they subsided almost as rapidly. The stimuli were transduced by a RCA $515 \mathrm{~S} 2$ loudspeaker, $1 \mathrm{~m}$ directly in front of the S.

The stimulus-generating equipment and recording system in the physiology laboratory have been described in Teas, Eldredge, and Davis (1962). A LINC computer has been added to control the stimulus presentation and the on-line data analysis. The signals were short-duration sinusoids with a three-wave rise, one-wave plateau, and three-wave fall. However, when the frequency of the stimulus was increased, none of the three time dimensions of the

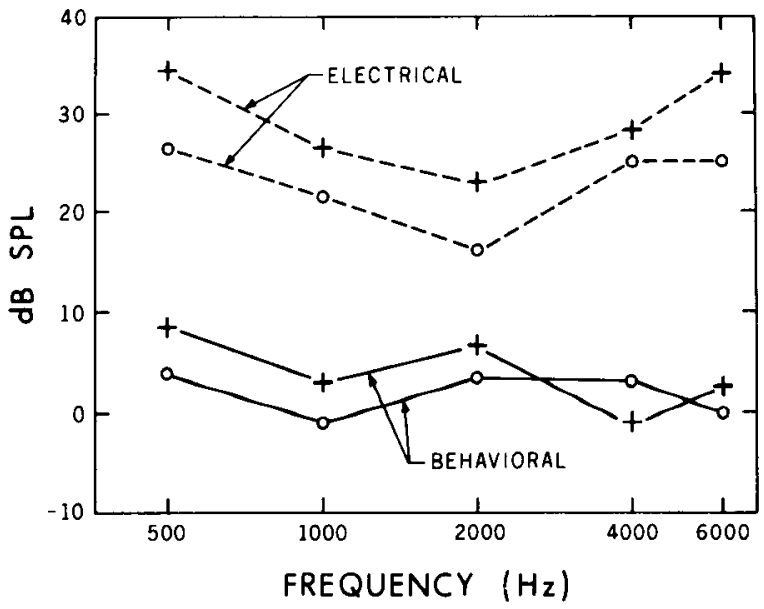

Fig. 3. The normal $(0 \ldots)$ and experimental $(+\ldots+)$ mean AER threshold curves are compared to the normal $(0-0)$ and experimental $(+-+)$ behavioral threshold curves. stimulus was ever shortened below 1 msec. The acoustic transducer was a 4-in. KLH Model 125 speaker. The chinchilla was restrained and the speaker was at $30 \mathrm{~cm}$ and 45 deg from the $S$ s head.

\section{AER Threshold Testing}

The AER thresholds are, in part, a function of the number of samples taken and the rate at which the stimuli are presented. In the experiments reported here the stimuli occurred every $250 \mathrm{msec}$ and the averaged AER was computed on 256 samples. This decision was a compromise to enhance the signal-to-noise ratio without making the data-collection time too long.

\section{Normal Groups}

The behavioral sensitivity functions for the experimental Ss were compared to the average sensitivity function of 36 normal chinchillas previously tested by J. D. Miller at the Central Institute for the Deaf. The AERs of the experimental animals with chronic electrodes are compared to either a group of eight anesthetized chinchillas who were tested with tone pips in the EEG Laboratory or a second group of eight anesthetized chinchillas who were tested with short-duration sinusoids in the Physiology Laboratory. The two normal AER groups were established during a process of screening the chinchillas for another experiment. Three chinchillas were rejected as not normal because of an elevated AER threshold. One of the three chinchillas was tested behaviorally and was found to have an elevated behavioral threshold, also.

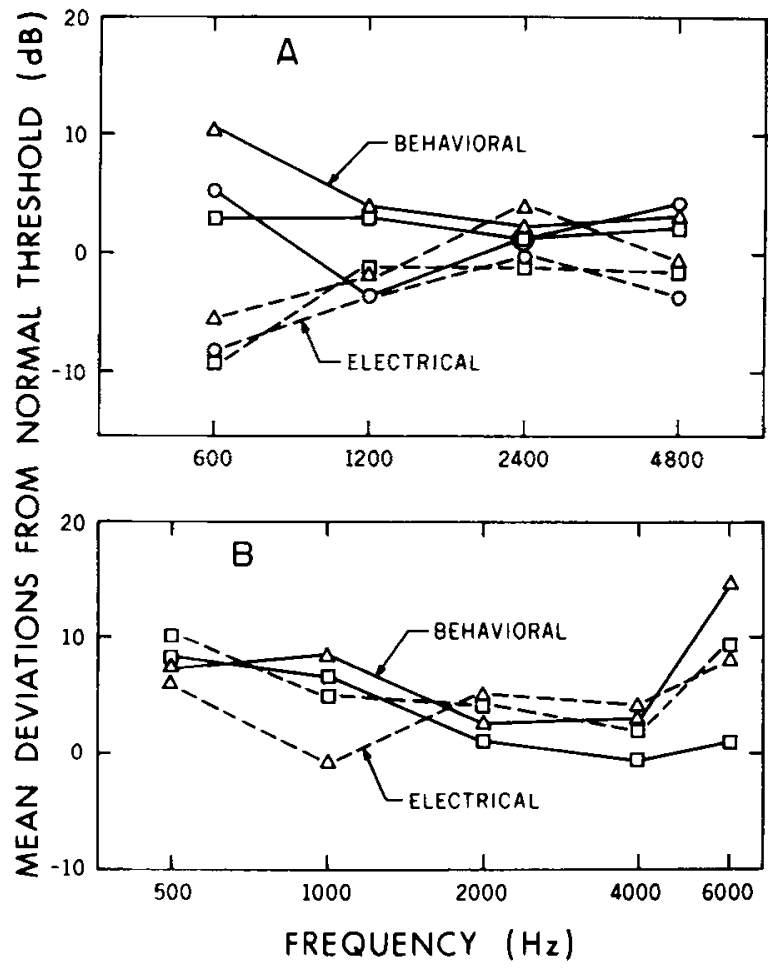

Fig. 4. Comparison of individual chinchilla AER ( . - ) and behavioral ( - ) thresholds over time. Chinchilla $202=\square$; Chinchilla $203=0$; Chinchilla 205 $=\triangle$. Each data point represents the deviation, in $\mathrm{dB}$, from the normal population threshold. Also, each data point is the mean of three successive days' testing. Data on Graph A were collected five weeks after the electrodes were placed on the skull. Data in Graph B were collected 10 weeks after the electrode placement.

\section{RESULTS}

AER from Awake and Anesthetized Chinchillas

In Fig. 2 individual early AERs recorded from conscious chinchillas with the chronic electrode assembly are compared to the AERs recorded from nembutal-anesthetized chinchillas using a 
subcutaneous electrode. The data were collected in the Physiology Laboratory using $1000 \mathrm{~Hz}$ signal with a $3-\mathrm{msec}$ rise, 1-msec plateau, and 3-msec fall-time. One cannot see any systematic difference between the groups. The early 8 -msec positive peak and later negative slow wave appear to be the same response that was recorded by Rothenberg and Davis (1967). There is a large amount of variability between $S s$ and across groups, but the general waveform and threshold for the response is approximately the same for awake and anesthetized Ss. Because there is no difference between responses from awake and anesthetized $S$ s it is possible to infer what the AER would be in a conscious state from recordings made under nembutal anesthesia.

\section{Comparison of Behavioral and AER Thresholds}

The mean AER and behavioral-response threshold curves for the experimental and normal groups are plotted in Fig. 3. The normal behavioral threshold curve is Miller's average of 36 chinchillas. The normal AER threshold curve is the average of eight chinchillas tested under nembutal anesthesia. The experimental threshold curves are the average of three chinchillas with the chronic electrode assembly. Both the AER and behavioral response thresholds of the experimental group are slightly higher than the thresholds for the normal groups. The behavioral threshold of both the normal and experimental group is 10 to $25 \mathrm{~dB}$ lower than the AER threshold. This 10 to $25 \mathrm{~dB}$ difference maly be the result of integration of the acoustic energy of the longer signals used in the behavioral test. Also, the ambient background noise level wils ligher in the AER testing chamber and the AER response may have been partially masked.

In Fig. 4 the individual AER and behavioral-response threshold curves are plotted as deviations from Miller's normal population values, i.e., in Fig. $4(\mathrm{~A})$, Chinchilla $205^{\prime}$ s AER $(\Delta-\Delta)$ threshold at $1200 \mathrm{~Hz}$ is $1.5 \mathrm{~dB}$ below normal and the behavioral threshold $(\Delta \Delta)$ is $3 \mathrm{aB}$ above nomal. The data show the individual variability and the degree of correlation between the two classes of response. The first series of three days of testing was conducted three weeks after the operation. Both the behavioral and AER thresholds were elevated approximately $15 \mathrm{~dB}$ above the population norm for all Ss. Otological examination revealed fluid in the middle ears of Chinchillas 202 and 205. Chinchilla 203 had a less severe infection. It is quite probable that the infection and accumulation of fluid accounted for the threshold elevation. The chinchillas were given Rondomycin for one week. One week after the Rondomycin therapy, all the ears were clear of infection. The chinchillas were tested again and the data are plotted in Fig. 4 (A). Both the behavioral and AER thresholds were normal. To check on the durability of the preparations, Chinchillas 203 and 205 were retested one month later. The data are plotted in Fig. 4 (B) and the thresholds are approximately normal.
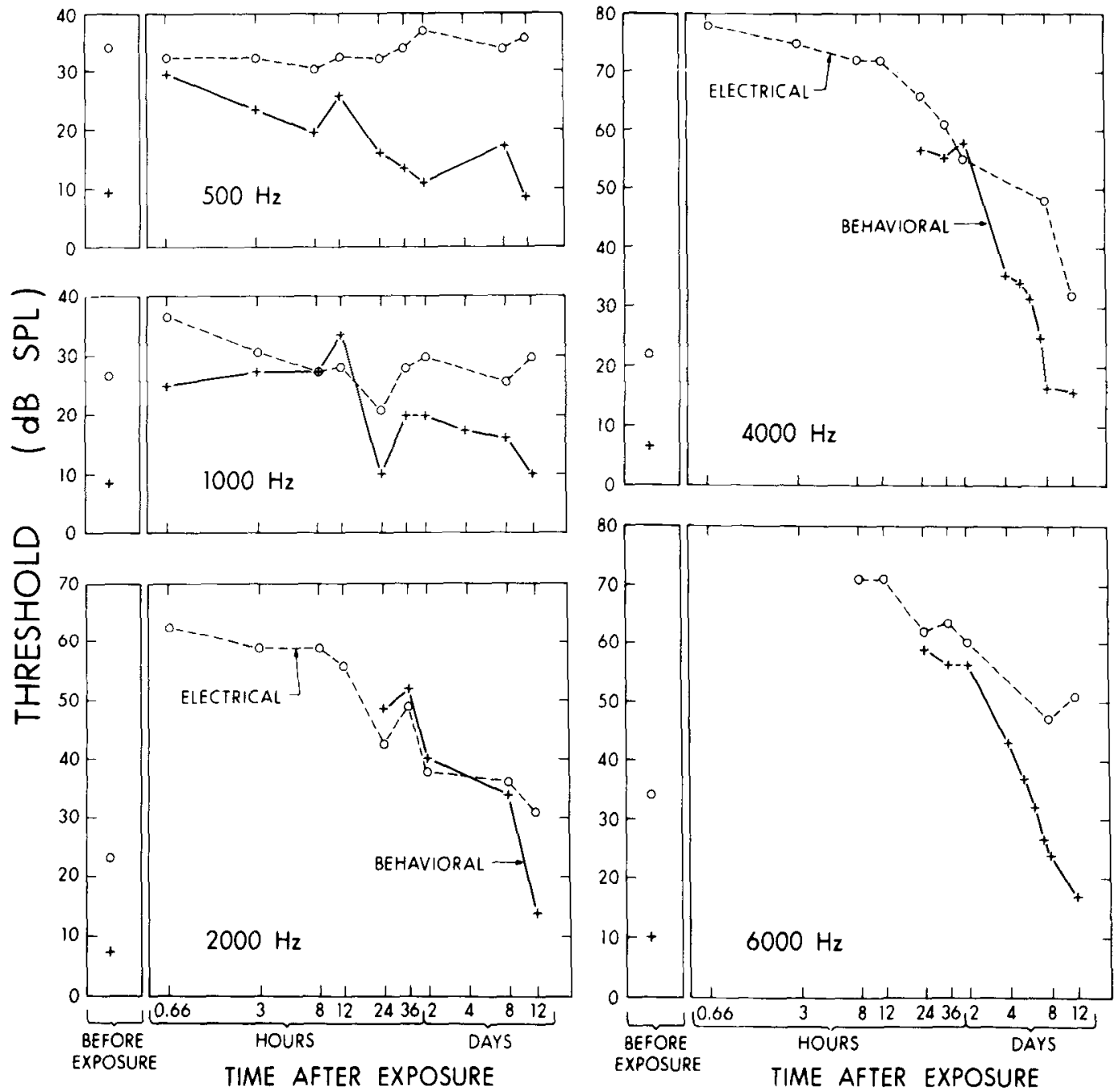

Fig. 5. Mean recovery curves for the AER and behavioral threshold after noise exposure. Note that the preexposure threshold is plotted at the left of each pair of recovery curves. 


\section{Noise Exposure}

Three chinchillas $(203,205,210)$ with chronic electrodes were exposed for $3 \mathrm{~h}$ to a $100 \mathrm{~dB}$ SPL octave band of noise centered at $2000 \mathrm{~Hz}$. The mean recovery curves for the various test frequencies are plotted in Fig. 5. The pre-exposure thresholds are plotted at the left of each graph. Forty minutes after exposure, thresholds are shifted only a few decibels at 500 and $1000 \mathrm{~Hz}$ but at 2000, 4000, and $6000 \mathrm{~Hz}$ the thresholds are shifted 40 to $60 \mathrm{~dB}$.

The 10 to $20 \mathrm{~dB}$ difference between the AER and behavioral threshold is reduced considerably after the noise exposure. The difference reappears and grows during the process of recovery. At 12 days both thresholds are normal for the low frequency stimuli but there is still a persistent AER and behavioral threshold shift at the two highest frequencies.

\section{Origin of the Early AER}

\section{DISCUSSION}

Rothenberg and Davis (1967) explored various nuclei in the brainstem of the chinchilla with a stainless steel electrode insulated to the tip. They found that the evoked response that could be recorded from the inferior colliculus matched the scalp response in both time and amplitude. If a response originates in the brainstem, then it is reasonable that it should be immune to general anesthetics.

In the present experiments, an attempt was made to record the AER from awake chinchillas with a needle electrode over the tentorium and a ground in the cheek pouch, but the noise level was too high in six of eight preparations. It appears that the mass of acrylic over the electrode assembly is necessary to insulate the electrodes from the muscle activity of a conscious animal and to stabilize the electrodes mechanically.

\section{AER and Behavioral Thresholds}

A first appraisal of Fig. 3 would lead to the conclusion that the chinchilla threshold for pure tones appears to be more sensitive when measured by the behavioral technique than by the AER method. However, one must consider the difference in the ambient noise level in the two test chambers and also the difference in the duration of the stimuli in the behavioral and the AER tests.

Using a Bruel and Kjaer Audio Spectrometer Model 2112, the spectral level of the noise was measured through the range of frequencies used as stimuli. It was found that the noise in the Physiology Laboratory was at least $5 \mathrm{~dB}$ greater than the noise in the Behavioral I aboratory (it is difficult to be precise because the noise in the Behavioral Laboratory was within the noise range of the measuring system). The AER thresholds measured in the Physiology Laboratory are masked thresholds. One can ask, how many decibels would the behavioral thresholds be elevated by the addition of a noise equal to the noise in the Physiology Laboratory? Given the spectral level of the noise through the range of frequencies tested, one can take the chinchilla critical ratio (CR), as measured by J. D. Miller and R. W. Gengel (1963) and estimate the masked threshold. The predicted masked threshold is equal to the spectral level of the noise plus the $C R$ in dB. The predicted masked threshold was found to be 5 to $8 \mathrm{~dB}$ higher than the normal chinchilla behavioral threshold. The large difference between the AER and behavioral thresholds might be reduced this 5 to $8 \mathrm{~dB}$ if the behavioral testing were done in an equally noisy test situation.

In man, the behavioral threshold is related to the duration of the stimulus, while the AER threshold is related primarily to the onset of the stimulus. Onishi and Davis (1967) have shown that the amplitude of the late human V-potential is dependent on only the first $30 \mathrm{msec}$ of the signal. It is our impression that the early AER recorded from over the tentorium of the chinchilla is even more dependent on the rise time of the stimulus. Presumably the voltage that is recorded arises from a population of neurons that fire more or less simultaneously and whose electrical output sums. As the stimulus continues in time, active neurons lose their synchrony and the net sum is a zero voltage. By contrast, psychophysical experinients have shown that the human $S$ behaves as an energy detector over the range from 30 to $250 \mathrm{msec}$. The signals used in the behavioral tests on the chinchillas were $750 \mathrm{msec}$. If the signals were reduced in duration to $6 \mathrm{msec}$, the mean duration of the signals used in AER testing, then it is reasonable to suppose that the behavioral thresholds would be elevated significantly. The difference between the two classes of thresholds would be reduced.

\section{Noise-Induced Threshold Shifts}

The two threshold curves did not shift proportionally after noise exposure. The behavioral threshold shift, $40 \mathrm{~min}$ after exposure, is approximately $15 \mathrm{~dB}$ at the two lowest frequencies and at least $60 \mathrm{~dB}$ at the three higher frequencies. The AER threshold shift, $40 \mathrm{~min}$ after exposure, is insignificant at the two low frequencies and is 25 to $45 \mathrm{~dB}$ at the three higher frequencies. The 15 to $25 \mathrm{~dB}$ difference between the AER threshold curve and the behavioral threshold curve is reduced or absent after noise exposure. The fact that the two classes of thresholds do not shift proportionally may be related to the way the ear integrates energy when there is a noise-induced threshold shift. Jerger (1955) showed that the human auditory system's ability to integrate energy over time is reduced after noise exposure. He exposed 12 human observers for $2 \mathrm{~min}$ to a $110 \mathrm{~dB}$ SPL thermal noise. The test stimuli were $4000 \mathrm{~Hz}$ sinusoids either 500,50 , or $5 \mathrm{msec}$ long. The pre-exposure thresholds for the 500 and $5 \mathrm{msec}$ signals were 15 and $29.1 \mathrm{~dB}$ SPL, giving a difference of $14.1 \mathrm{~dB}$. Two minutes after exposure the threshold for the $500 \mathrm{msec}$ signal was $27 \mathrm{~dB}$ $S P L$ and the threshold for the $5 \mathrm{msec}$ signal was $33.9 \mathrm{~dB}$. The difference between the 500 and $5 \mathrm{msec}$ signal had been reduced from $14.1 \mathrm{~dB}$ to $6.9 \mathrm{~dB}$

The chinchillas received a much more traumatic exposure than Jerger's Ss, i.e., the human Ss had a $5-\mathrm{dB}$ shift for the 5-msec signal 12 min after exposure and the chinchillas had a $50-\mathrm{dB}$ shift in their behavioral threshold $12 \mathrm{~h}$ after the exposure. The dependence of the behavioral threshold on the duration of the stimulus is reduced considerably when the auditory system is traumatized by noise exposure. One can hypothesize that the convergence of the two classes of threshold curves is caused by the disruption of the auditory system's ability to integrate energy, and the long signals in the behavioral test are processed like short signals.

In an experiment by Henderson (in press), two chinchillas were trained to respond to $2000 \mathrm{~Hz}$ signals of durations varying from 25 to $750 \mathrm{msec}$. The threshold for the $25 \mathrm{msec}$ signal is $12 \mathrm{~dB}$ higher than the threshold for the $750 \mathrm{msec}$ signal. The chinchillas were given the same noise exposure as described above. Four minutes after exposure the threshold for the $25-\mathrm{msec}$ signal was elevated $55 \mathrm{~dB}$ and the threshold for the $750-\mathrm{msec}$ signal was elevated $65 \mathrm{~dB}$. The larger shift for the 750 -msec signal erased the difference between the 25 - and $750-\mathrm{msec}$ thresholds. However, the difference reappeared during the process of recovery.

\section{CONCLUSIONS}

An AER can be recorded from an awake chinchilla with a chronic stainless-steel electrode placed on the dura over the tentorium. This response is virtually identical with the response that can be recorded from a subcutaneous electrode placed over the tentorium of a nembutal-anesthetized chinchilla. Presumably, the responses are generated in the inferior colliculus. The electrical response is an early positive wave with a peak of 6 to $8 \mathrm{msec}$ after the stimulus onset. A second, more variable, negative wave follows the first wave and has a latency of approximately 12 to $18 \mathrm{msec}$ after the stimulus onset.

When short signals were used, the behavioral and AER response had essentially the same threshold. The behavioral response appears at lower levels for longer signals and is consistent with temporal integration data for humans. The AER is complete in a short time and is clearly an onset phenomenon.

In the presence of middle ear infection and following high level exposure to noise, both the AER and the behavioral response 
thresholds were elevated. Numerically, these threshold shifts covaried in a manner consistent with our understanding of the process of temporal integration.

\section{REFERENCES}

DAVIS, H., MAST, T., YOSHIE, N., \& ZERLIN, S. The slow response of the human cortex to auditory stimuli: Recovery process. Electroencephalography \& Clinical Neurophysiology, 1966, 21, 105.113.

DAVIS, H., HIRSH, S. K., SHELNUTT, J., \& BOWERS, C. Further validation of evoked response audiometry (ERA). Journal of Speech \& Hearing Research, 1967, 10, 717.732.

HENDERSON, D. Temporal integration in the chinchilla. Journal of the Acoustical Society of America. In press.

JERGER, J. Influence of stimulus duration on the pure-tone threshold during recovery from auditory fatigue. Journal of the Acoustical Society of America, 1955, 27, 121-125.

MILLER, J. D., WATSON, C. S., \& COVELL, W. P. Deafening effects of noise on the cat. Acta Oto-Laryngologica-Supplement 176, 1963.

PETERS, E. N. Temporary shifts in auditory thresholds of chinchillas after exposure to noise. Journal of the Acoustical Sociely of America. 1965, 37, $831-833$.
ROTHENBERG, S., \& DAVIS, H. Auditory evoked response in chinchilla: Application to animal audiometry. Perception \& Psychophysics, 1967,2, 443-447.

TEAS, D. C., ELDREDGE, D. H., \& DAVIS, H. Cochlear responses to acoustic transients: An interpretation of whole-nerve action potentials. Journal of the Acoustical Society of America, 1962, 34, 1438-1459.

\section{NOTES}

1. This investigation was supported by Public Health Service Research Grant NB03856 from the National Institute of Neurological Diseases and Blindness.

2. Address: Contral Institute for the Deaf, 818 South Euclid. St. Louis, Mo. 63110.

3. N1H International Posidochal fellow

(Accepted for publication September 10, 1968.) 